\title{
Proliferation IMPAIRS CELL VIABILITY VIA ENERGY DEPLETION
}

Pierre Galichon ${ }^{1,2,3^{*}}$, Morgane Lannoy ${ }^{2}$, Li $\mathrm{Li}^{1}$, Sophie Vandermeersch ${ }^{2}$, David Legouis ${ }^{4}$, Michael T Valerius ${ }^{1}$, Juliette Hadchouel ${ }^{2}$ and Joseph V Bonventre ${ }^{1}$

1. Department of Medicine, Harvard Medical School, and Renal Division, Department of Medicine, Brigham and Women's Hospital, Boston, MA, USA

2. INSERM UMRS_1155, France

3. Sorbonne Université, APHP, AP-HP. Sorbonne Université, F-75020 Paris, France.

4. Laboratory of Nephrology, Department of Medicine and Cell Physiology, Division of Intensive Care, University Hospital of Geneva, Geneva, Switzerland.

* Corresponding author: Pierre Galichon.

Inserm 1155, Bâtiment Recherche Hôpital Tenon

4 rue de la Chine 75020 Paris, FRANCE

Tel.: +33685717580Ｅmail: pierre.galichon@aphp.fr 


\section{ABSTRACT}

Introduction. Proliferation is essential to the development of all living organisms and to the replacement of dead cells in injured organs. Proliferation is tightly regulated, and loss of proliferation control is a hallmark of cancer. Thus, cell proliferation and cell survival are closely interconnected to contribute to organ homeostasis or tumor development. Given the central role of energy homeostasis in cell survival, and the fact that proliferation increases negentropy, we hypothesized that proliferation might affect the intracellular ATP/ADP ratio, a robust readout of the cellular energy status.

Methods. We analyzed transcriptomic data and cytotoxicity assessment from tumor cell lines challenged with a panel of chemotherapies. We then analyzed the effect of proliferation on the viability and on the intracellular ATP/ADP ratio of epithelial cell lines challenged with toxic or energetic stresses. Finally, we studied transcriptomic data from both tumors and injured or recovering kidneys, and computed indexes for proliferation, and nuclear vs mitochondrially encoded oxidative phosphorylation genes.

Results. Here we found that proliferation is associated with decreased survival after toxic or energetic stresses in both cancer and epithelial cells. In vitro, we found that ATP/ADP ratio was tightly regulated throughout the cell cycle, and that proliferation was instrumental to an overall decrease in intracellular ATP/ADP ratio. In vivo, we found that the expression of genes of the oxidative phosphorylation pathway (OXPHOS) was correlated with proliferation in cancer. In injured kidneys, proliferation was also associated with increased expression of genes of the oxidative phosphorylation pathway encoded in the nucleus, but mitochondrially-encoded genes were strongly decreased, suggesting the coexistence of a passive mitochondrial injury and an adaptative nuclear response with opposite effects on OXPHOS. Increased proliferation and decreased expression of mitochondrially-encoded genes of the oxidative phosphorylation pathway were associated with a poor renal outcome. In sum, we show that proliferation is an energy demanding process impairing the cellular ability to cope with a toxic or ischemic injury. 


\section{INTRODUCTION}

Life can be defined as a self-sustained limitation of entropy (negentropy) (Roger et al., 1992). Proliferation is often included in the definitions of life as it pertains both to sustaining a life form and to limiting entropy by increasing the number of energy-containing entities. As such, proliferation requires energy. Proliferation is tightly controlled in normal and pathological situations. Proliferation is the cornerstone of development in multicellular organisms, yet, it is finely orchestrated, and various stresses in prokaryotes or multicellular organisms trigger pathways inhibiting proliferation, suggesting that uncontrolled proliferation can be deleterious, not only in cancer (Gordan et al., 2007; Motoshima et al., 2006). On the other hand, proliferation is an important aspect of the repair process following injury (Price et al., 2004).

Adenosine triphosphate (ATP) is the main energy source for intracellular processes, through its cleavage into adenosine diphosphate (ADP) and hydrogen phosphate (Veech et al., 1979; Bonora et al., 2012). Because ATP is present in every cell, its concentration is strongly correlated with cell density. ATP concentration is therefore used as a marker to monitor the cell number (Crouch et al., 1993). On the other hand, the intracellular ATP/ADP or ATP/AMP ratio is a more reliable marker of the cell's energy status (Maldonado and Lemasters, 2014). ATP/ADP and ATP/AMP are equivalent as $2 \mathrm{ADP}$ are converted to $1 \mathrm{AMP}+1 \mathrm{ATP}$ in a null enthalpy reaction (Dixon, 1988). Genetically encoded markers of the ATP/ADP ratio have been developed, allowing its intracellular monitoring in live cells, and thus providing a reliable marker of the cell's energy status (Berg et al., 2009; Tantama et al., 2013).

High cellular energy is generally accepted as a marker of viability (Chan et al., 2013) and is considered a prerequisite for cell proliferation (Fu et al., 2016; Wang et al., 2017). Conversely, a persistent decrease in the level of intracellular ATP is associated with cell death, both in tumor and non-tumor cells (Lelli et al., 1998; Verrax et al., 2011). It is generally assumed that the 
ATP/ADP ratio is regulated prior to changes in proliferation (Marcussen and Larsen, 1996; ShollFranco et al., 2010; Song et al., 2010; Hardie, 2011). Indeed, a low ATP/ADP ratio (or its close correlate, ATP/AMP) activates AMPK, which induces cell cycle arrest through p27, p53 and p21 activation in various organs (Hardie et al., 2012). By contrast, in cancer, a low ATP/ADP ratio is considered to allow cell proliferation by stimulating aerobic glycolysis and the Warburg effect (Maldonado and Lemasters, 2014). Although the effect of ATP/ADP variations on proliferation has been studied, the reverse mechanism, i.e. the effect of cell cycle progression on the cellular energy status, is yet unexplored. Because the ATP/ADP ratio reflects the energy in a cell (Atkinson, 1968; Vander Heiden et al., 2009; Tarasov et al., 2012), and energy depletion leads to cell death (Izyumov et al., 2004; Verrax et al., 2011), we hypothesized that the effect of proliferation on the $\mathrm{ATP} / \mathrm{ADP}$ ratio might impact the cellular fate more strongly than the regulatory feedback on proliferation triggered by changes in the ATP/ADP ratio. Here, using viability assays and transcriptomic analyses, we investigate the relationship between proliferation and viability in human cancer cells and human epithelial cells. By quantifying the ATP/ADP ratio in live cells using a single cell approach, we study the effect of proliferation on the intracellular ATP/ADP ratio, and characterize the pattern of variation of the energy level throughout the cell cycle. Lastly, we investigate the differential transcriptomic signature of energy production in proliferating cancer cells and in proliferating epithelial cells after injury, highlighting the contrasted consequences of proliferation in tumoral versus non-tumoral diseases on energy homeostasis and cell viability. 


\section{RESULTS}

\section{Proliferating cells are more sensitive to an injury}

We studied the consequences of cell proliferation on cell viability in vitro. In the GDSC database, the chemosensitivity of 1021 cancer-derived cell lines was assessed for 367 different molecules (Yang et al., 2013). We found a significant correlation $(\mathrm{p}<0.05)$ of the proliferation index with chemosensitivity for 257 molecules $(70 \%[65-75]$, p-value=1.1e-14). The correlation was positive for $90 \%[86-94]$ of them (p-value=2.2e-16) (Figure $1 \mathrm{~b}$ and $\mathrm{d}$ ). It is interesting to note that chemosensitivity was most associated to proliferation for drugs targeting cell cycle, metabolism and apoptosis, whereas chemoresistance was most associated with proliferation for MEK1/2 inhibitors (Suppl Table 1).

This analysis suggests that proliferation impairs the cell ability to cope with an injury. In order to test this hypothesis in non-cancer cells, renal epithelial cells (HK2 cell line) were subjected to a toxic or metabolic stress when their proliferation was either stimulated by pifithrin$\alpha$ (a p53 antagonist promoting G1/S and G2/M transition) or inhibited by tenovin-1 (a p53 agonist inhibiting the G1/S transition) (Figure 1a). We observed that the stimulation of cell proliferation by pifithrin- $\alpha$ promoted the death of the cells exposed to puromycin (a well characterized toxicant acting through inhibition of protein synthesis), whereas inhibition of cell proliferation by tenovin-1 was protective. Pifithrin- $\alpha$ or tenovin-1 alone were not toxic by itself, as they did not cause cell death in the absence of puromycin (Figure 1a). The same result was obtained when cells were cultured under conditions of chemical hypoxia without glucose versus control conditions (normoxia + glucose) (Figure 1c). Taken together, these data show that proliferation impairs the ability of cells to survive an injury. Given that proliferation requires 
energy and that a decreased cellular energy level results in cell death, we hypothesized that the depletion of cellular energy could be responsible for this impairment.

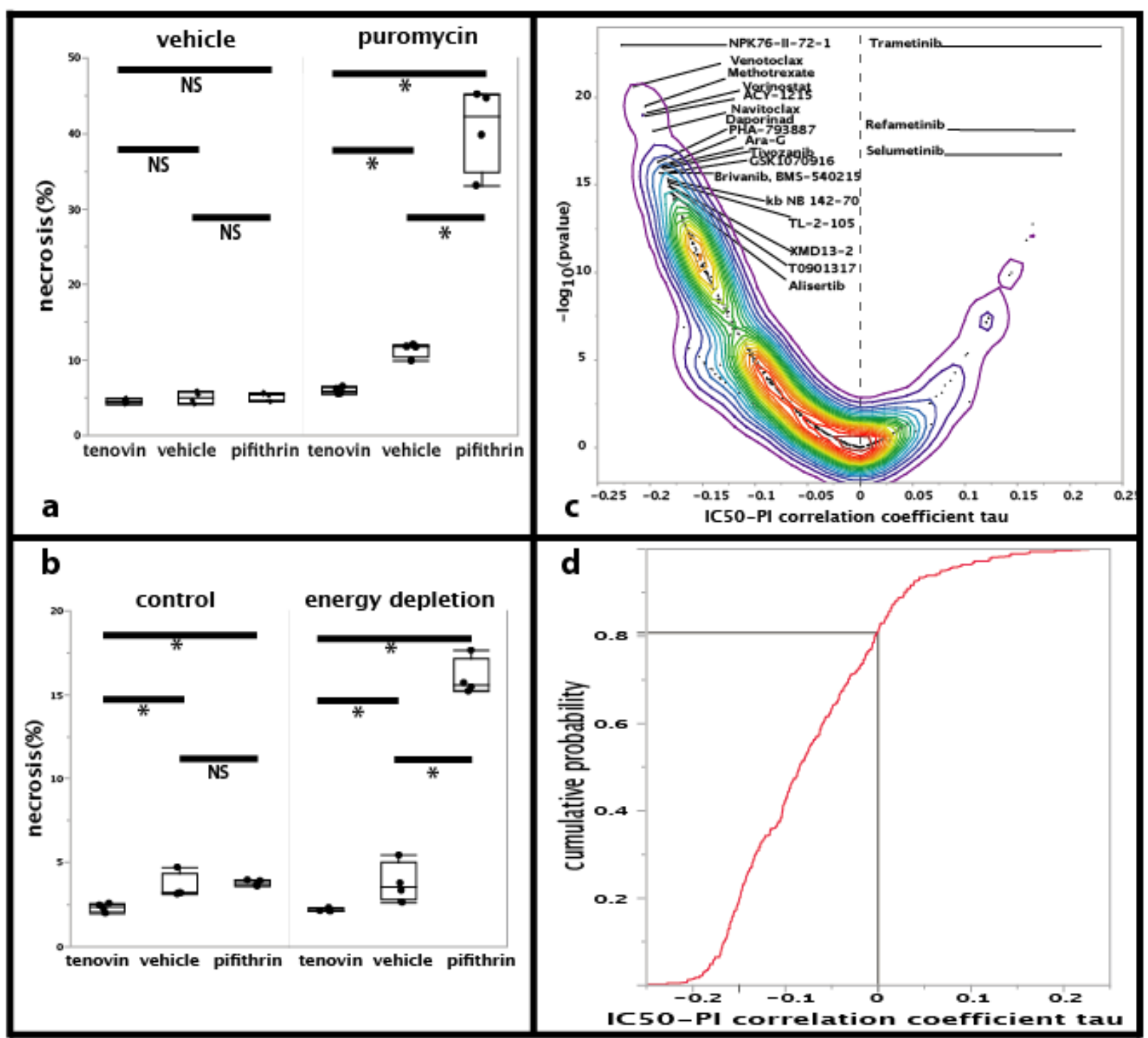

Figure 1: Consequences of cell proliferation on susceptibility to cell death. a. Effect of cell cycle inhibition by tenovin-1 or cell cycle facilitation by pifithrin- $\alpha$ on cellular death caused by puromycin. b. Effect of cell cycle inhibition by tenovin-1 or cell cycle facilitation by pifithrin- $\alpha$ on cellular necrosis caused by energy depletion (oxygen and glucose deprivation). c. Vulcano plot of the correlation of the cytotoxicity index with the proliferation index tested for 367 compounds. The top 20 significant correlations are labeled. d. Cumulative probability for all 367 compounds is plotted against the coefficient of the correlation between IC50 and proliferation index, showing a skewed distribution towards correlation between proliferation and chemosensitivity *: p-value $<0.05$; NS: statistically non-significant. 


\section{The ATP/ADP ratio decreases when cells proliferate}

In order to follow the cellular energy level during the course of a cell cycle, we measured the ATP/ADP ratio in proliferating human renal epithelial cells (HK2 cell line). PercevalHR is a genetically encoded fluorescent marker which enables the live monitoring of the ATP/ADP ratio (Tantama et al., 2013; Berg et al., 2009). To monitor the single cell-ATP/ADP ratio over time in proliferating epithelial cells, we generated human renal epithelial HK2 cells stably expressing PercevalHR (Suppl Figure 1a). We first validated this quantification of the ATP/ADP ratio (ATP/ADP[Perceval]) by an independent technique based on the measurement of luciferin/luciferase bioluminescence (ATP/ADP[Luciferin]). The total ATP/ADP[Perceval] signal correlated well with the ATP/ADP[Luciferin] $(\mathrm{r}=0.96, \mathrm{p}=0.0001)$. We also observed the expected decrease of ATP/ADP[Perceval] after chemical energy depletion with blockage of glycolysis and oxidative metabolism using 2-deoxyglucose (2 DG) and sodium azide $\left(\mathrm{NaN}_{3}\right)$ (Suppl Figure $1 \mathrm{~b}$ and 1c).

Single cell ATP/ADP[Perceval] in real-time was quantitated in vitro. Although changes in the mean ATP/ADP values over time were reproducible in basal conditions or during energy depletion, this ratio was highly variable among proliferating cells in the same well and at the same time (Suppl Figure 1c). This observation suggests that the ATP/ADP ratio is a dynamic parameter, undergoing variations that are at least partly independent of the extracellular environment. We hypothesized that the ATP/ADP ratio could be correlated with the cell cycle stage. To test this hypothesis, we plated PercevalHR-expressing epithelial cells at various densities to achieve different levels of contact inhibition of proliferation (Figure 1a, left panel). We found that the intracellular ATP/ADP ratio increased when the cell density was higher. To rule out a direct effect of the confluence, we cultured the cells at the same densities in the presence of rigosertib, a potent cell cycle inhibitor inducing G2/M arrest. Non-proliferating cells 
showed a strong increase in the ATP/ADP ratio, regardless of their degree of confluence. Conversely, performing a scratch assay in confluent cells induced the proliferation and migration of the cells, which then displayed a low ATP/ADP ratio. Again, rigosertib increased the cellular ATP/ADP ratio while inhibiting wound closure (Figure 1a, right panel).

To exclude a possible cell cycle-independent effect of rigosertib, we then used a panel of pharmaceutical compounds to study the changes in the ATP/ADP ratio over time when the cell cycle is perturbed (Figure 1b and 1c). Tenovin-1 (P53 agonist) and Rigosertib (PLK1 inhibitor) were used to inhibit the cell cycle during the G1/S or G2/M transition, respectively. Conversely, pifithrin- $\alpha$ (P53 antagonist promoting G1/S and G2/M transition) and KU-55933 (ATM inhibitor promoting G2/M transition) were used to stimulate cell proliferation. Single-cell monitoring demonstrated that cell cycle inhibition increased ATP/ADP progressively over time whereas cell proliferation decreased it (Figure 1c). 


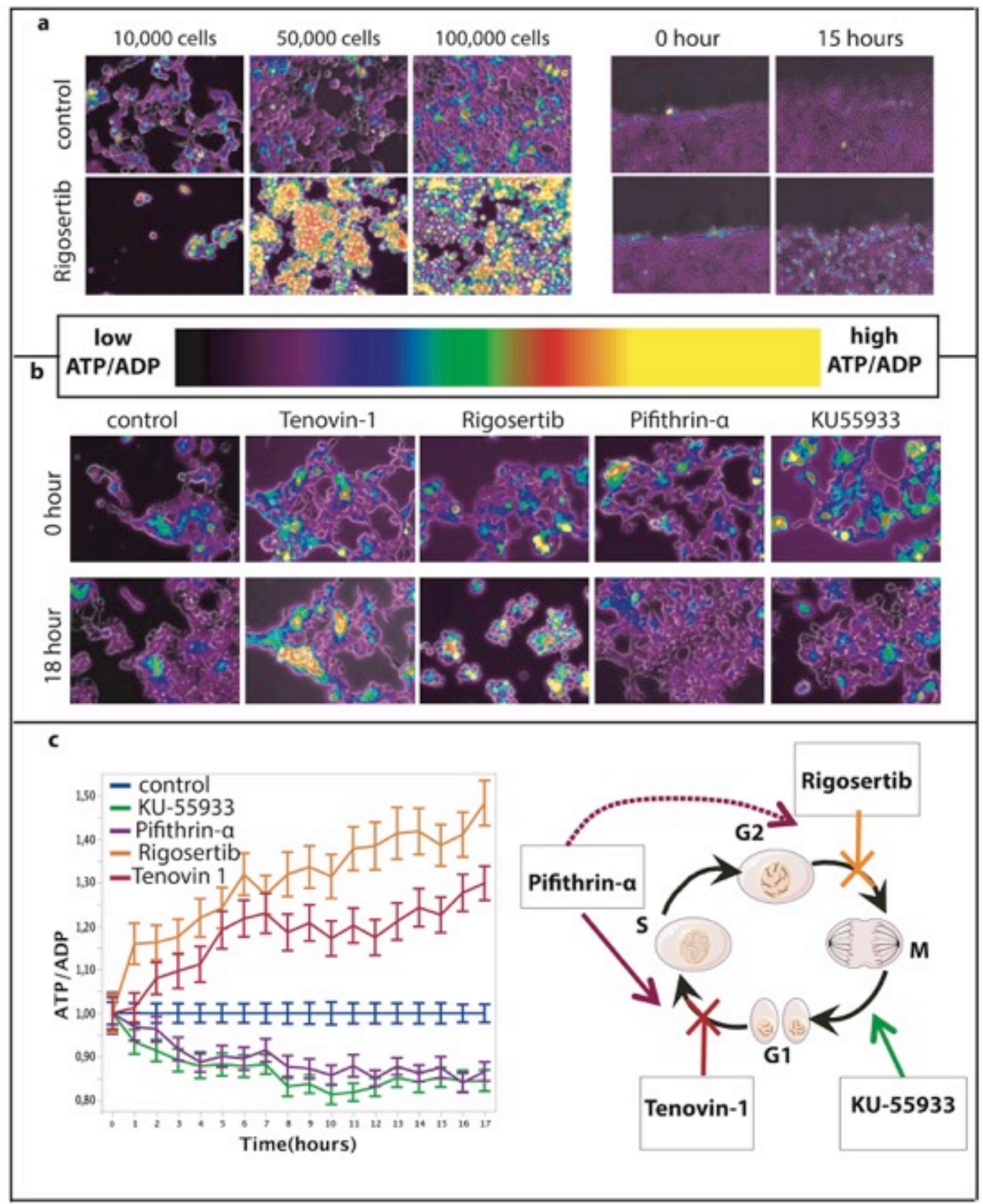

Figure 1: Cell cycle interventions and their effects on ATP/ADP ratio.

a. Wells were plated with increasing numbers of PercevalHR expressing cells to obtain various levels of confluence in control condition or with $1 \mu \mathrm{M}$ rigosertib for cell cycle inhibition. A scratch assay was performed under control condition or with exposure to $1 \mu \mathrm{M}$ rigosertib to inhibit wound closure, shown 0 and 15 hours after the scratch. b. Pharmaceutical interventions on the cell cycle using various compounds on PercevalHR expressing cells over an 18-hr time course: KU55933 $(10 \mu \mathrm{M})$, pifithrin- $\alpha(50 \mu \mathrm{M})$, rigosertib $(10 \mu \mathrm{M})$, tenovin-1 $(10 \mu \mathrm{M})$. c. Quantification of changes in single cell intracellular ATP/ADP ratio over an 18-hr exposure to various cell cycle modifiers. 


\section{Cell cycle and proliferation-dependent variations in the intracellular ATP/ADP ratio}

The single-cell ATP/ADP ratio was maximal around cytokinesis in non-synchronized cells (Figure 2a, left panel). Variations of the ATP/ADP ratio before and after cytokinesis follow a systematic pattern (Figure 2a, right panel): after a gradual increase, the ratio peaks at mitosis before dropping dramatically. This pattern was highly reproducible, including in cells exposed to pharmaceutical inhibition or stimulation of the cell cycle (Figure $2 b$ ). The cell cycle inhibitor tenovin-1 increased the mean ATP/ADP ratio over time compared to the cell cycle facilitator pifithrin- $\alpha$ (Figure 2b, left panel). Thus, the intracellular energy level, evaluated by the ATP/ADP ratio, is directly influenced by the cell cycle.

In order to study potential variations in the single cell ATP/ADP ratio in successive cell cycles within the same cell, ATP/ADP ratio trajectories were evaluated in cells undergoing division twice in the same live-imaging experiment. After a first mitosis, cells treated with tenovin-1 increased their ATP/ADP ratio significantly more than cells treated with pifithrin- $\alpha$ (Figure 2b, upper right panel). The cells had the same pattern of ATP/ADP variations for each of the two successive mitoses. In cells treated with tenovin-1, there was an increment in the mitotic maxima of the ATP/ADP ratio from one mitosis to the next, whereas such an increment did not occur with the cell cycle facilitator pifithrin- $\alpha$ (Figure $3 b$, bottom right panel). We conclude that cell cycle inhibition increases ATP/ADP ratio in cells independently of the cell cycle stage, in a time dependent manner: the slower the cell cycles, the more positive is the cellular energy balance. 


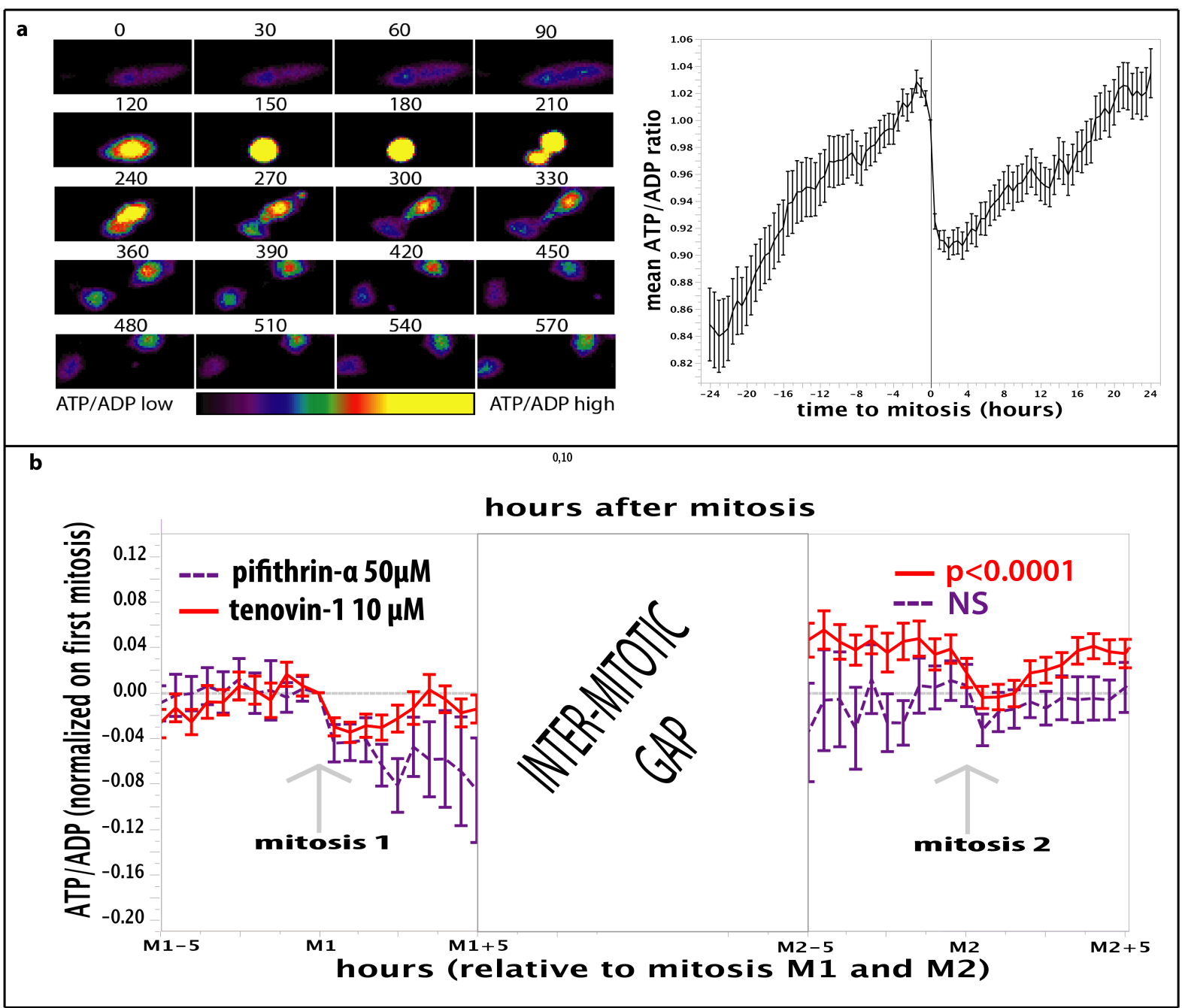

Figure 2: ATP/ADP variations throughout the cell cycle.

a. Live imaging on a single PercevalHR expressing cell undergoing mitosis (left). Quantification of the intracellular ATP/ADP ratio in single cells during the peri-mitotic period with in silico synchronization on time of mitosis (right). b. ATP/ADP changes in PercevalHR expressing cells incubated with the cell cycle facilitator pifithrin- $\alpha$ or with the cell cycle inhibitor tenovin-1, over the whole experiment (left), after the first mitosis (upper right panel) and between 2 successive mitoses (lower right panel). ATP/ADP ratio values are represented as means and whiskers indicate the standard error. P-values represent the significance of the comparison between the first and second mitosis within each treatment group, using a Wilcoxon rank test. 


\section{Correlation between proliferation and the transcriptome of energy metabolism in vivo}

To test the relevance of our findings in vivo, we studied the transcriptomic profiles of 8375 tumors from the The Cancer Genome Atlas (TCGA) database and of ischemic kidneys before and after transplantation (Cippà et al., 2018). We calculated the proliferation index to estimate the proliferation index, as previously described (Venet et al., 2011). Pathway analysis was based on the correlation of each gene expression profile with the proliferation index, with focus on the three energy-producing pathways from the Kyoto Encyclopedia of Genes and Genomes (KEGG) :oxidative phosphorylation, citrate cycle, and glycolysis/neoglucogenesis (Kanehisa and Goto, 2000). We found that the level of expression of the genes involved in ATP generation (oxidative phosphorylation,) was strongly associated with proliferation in both cancers and ischemic kidneys (Table 1 and Suppl Table3). The directional analysis showed that the proliferation index was positively correlated with the level expression of genes involved in oxidative phosphorylation in cancers, but negatively correlated in ischemic kidneys. Within the group of transplanted kidneys, three evolutive profiles were studied separately: recovery, transition towards chronic kidney disease (transitional) and established chronic kidney disease (CKD) (Cippà et al., 2018). A subgroup analysis showed that oxphos downregulation was consistently associated with proliferation in the three evolutive profiles (Suppl Table 3). 


\begin{tabular}{|l|l|l|}
\hline pathway & TCGA OR (p value) & reperfusion OR (pvalue) \\
\hline Oxphos & $\mathbf{1 . 0 0 7}(\mathbf{1 . 1 3 e - 1 0 )}$ & $\mathbf{0 . 5 4 2}(\mathbf{2 . 7 5 e - 2 2 )}$ \\
\hline TCA & $1.002(4.89 \mathrm{e}-1)$ & $\mathbf{0 . 7 0 0}(\mathbf{7 . 6 3} \mathrm{e}-4)$ \\
\hline Glycolysis & $1.001(5.70 \mathrm{e}-1)$ & $0.869(7.61 \mathrm{e}-2)$ \\
\hline
\end{tabular}

Table 1. Association of differentially expressed genes with Energy metabolism pathways from the KEGG encyclopedia, performed on cancer samples from the TCGA repository and on kidneys immediately after transplantation.

\section{The expression of the OXPHOS genes encoded in the mitochondria is decreased upon injury}

A further analysis showed that the decrease of OXPHOS genes expression with proliferation in ischemic kidneys is actually caused by a profound downregulation of the mitochondria-encoded genes. In contrast, the nucleus-encoded genes associated with oxphos were moderately upregulated with proliferation (Figure 3). In tumors, the proliferation index was positively correlated with the expression level of both mitochondria- and nucleus-encoded genes (Figure 3).

In transplanted kidneys, persistent proliferation at 3 months or 1 year after transplantation significantly correlated with decreased oxidative phosphorylation and with chronic kidney disease as evidenced by decreased glomerular filtration rate, increased renal fibrosis and fibrosis progression (Figure 4 and Supplemental Figure2). Interestingly, oxphos transcripts were found to be downregulated in the nucleus during the acute phase of injury (reperfusion), and downregulated in the mitochondria at the chronic phase of transition do chronic kidney disease (Figure 4).

This suggests that proliferation is always associated with an increased expression of oxidative phosphorylation-related genes in the nucleus, but that it can be dissociated from mitochondriallyencoded genes in case of injury, which could represent a limiting factor for energy production. 


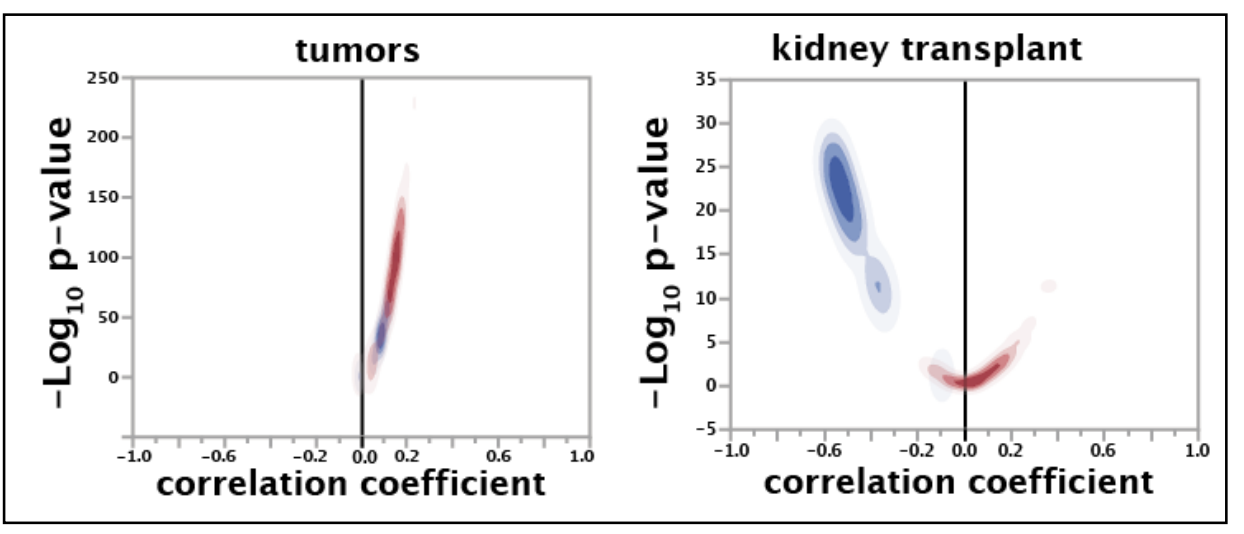

Figure 3. Correlation of OXPHOS gene expression with the proliferation index in cancers (left panel) and in ischemic kidney(right panel). x-axis: Kendall's k coreelation coefficient; y-axis: significance represented by the - $\log$ (pvalue) of the correlation (Kendall); blue: mitochondrially encoded genes; red: nucleus-encoded genes.

\section{Association of proliferation, mitochondrial status and outcomes.}

The proliferation index was higher in the kidney biopsies performed 3 or 12 months after transplantation versus biopsies performed immediately before or after transplantation. The OXPHOS index did not differ between these different time points, except for a decrease in overall OXPHOS index immediately after transplantation (Figure 4 a). More significantly, the proliferation index and the mitochondrial OXPHOS index were different in the 3 different evolutive patterns (recovery, transitional and CKD), a high proliferation index and a low mitochondrial OXPHOS index being associated with chronic disease. Interestingly, the overall OXPHOS index, mostly dependent on nucleus encoded OXPHOS genes, was not different among these groups (Figure $4 \mathrm{~b}$ ). When considering long term clinical outcomes, we found that the timing dramatically modified the relationship between of proliferation and OXPHOS and the incidence of fibrosis in the kidney (Figure $4 \mathrm{c}$ and Supplemental Figure 2). Low mitochondrial OXPHOS index and high proliferation index in late biospies (3 or 12 months) was associated with increased fibrosis between 3 and 12 months after transplantation. On the opposite, low mitochondrial OXPHOS in the early biopsies (immediately before and after transplantation) was 
associated with less incidence of fibrosis in the kidney between 3 and 12 months after transplantation, and the proliferation index did not correlate in the early biospies with changes in kidney fibrosis (Figure $4 \mathrm{c}$ ). All time points taken together, in the transplanted kidneys, the proliferation index was positively correlated with the overall OXPHOS index, but negatively correlated with the mitochondrial OXPHOS index. 


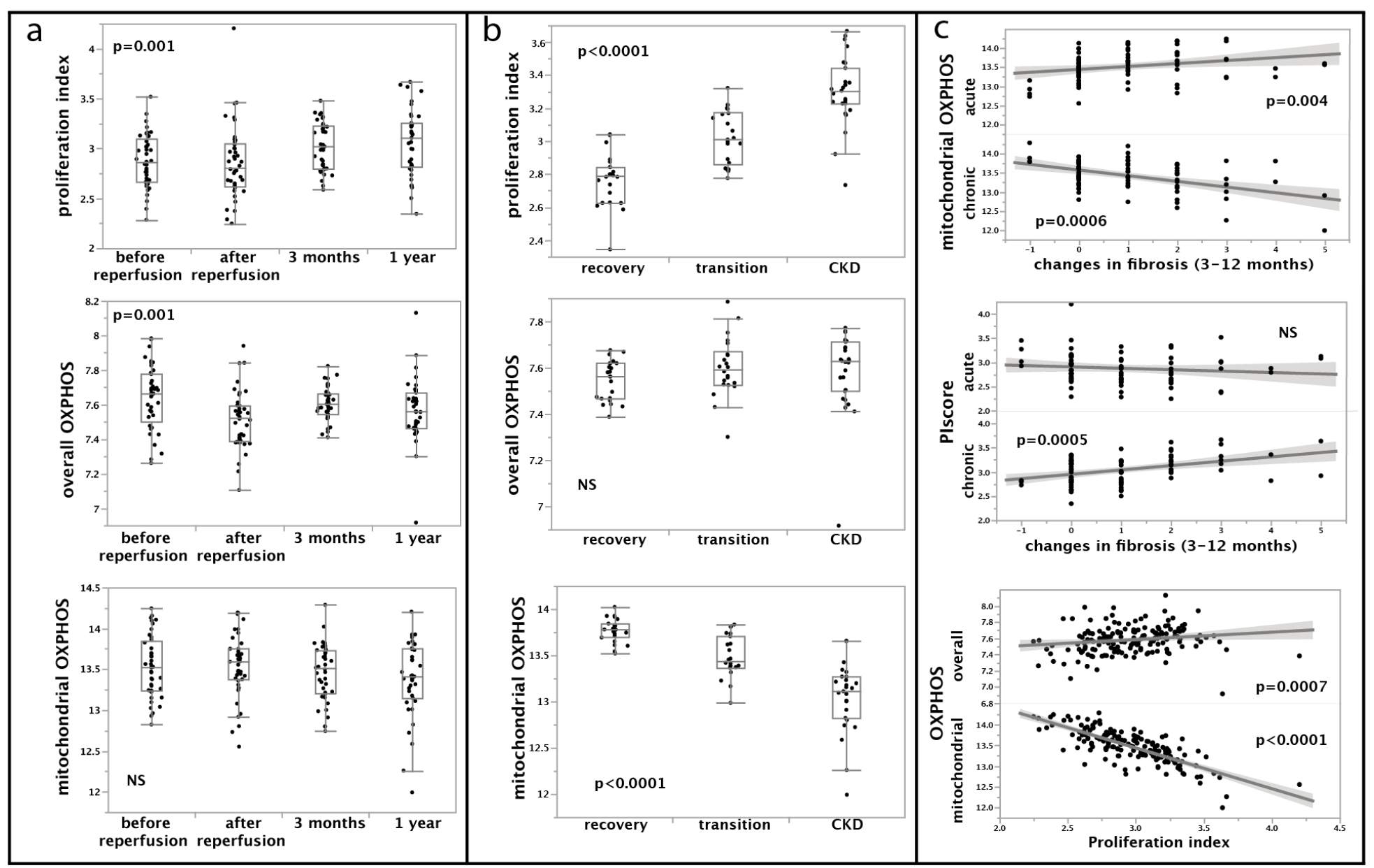

Figure 4 Association of proliferation index and OXPHOS with chronic kidney disease. a. Proliferation index, OXPHOS index and mitochondrial OXPHOS index among different evolutive profiles (recovery, transition to chronic kidney disease, chronic kidney disease (CKD). b Proliferation index, OXPHOS index and mitochondrial OXPHOS index at different times after ischemic injury (before transplantation, after reperfusion, 3 months after transplantation, 1 year after transplantation). c. Mitochondrial OXPHOS and proliferation index at acute (before transplantation and after reperfusion) and chronic time points (at 3 and 12 months after transplantation) differential correlations with fibrosis progression between 3 and 12 months, and the differential correlations of overall and mitochondrial OXPHOS with the proliferation index. 


\section{DISCUSSION}

\section{Proliferation and energy metabolism are interdependent}

The fact that low intracellular ATP induces molecular pathways downstream of AMPK to control proliferation suggests the presence of a regulatory feedback to mitigate the decrease in intracellular ATP levels caused by increased energy consumption. Such an effect of proliferation on ATP could explain why an increase in cell proliferation aggravates the course of acute kidney injury caused by an ischemic episode (Price et al., 2004), and why highly proliferative tumors frequently undergo spontaneous necrosis (Verrax et al., 2011). The kidney has a very high energy turnover and is thus very sensitive to injury (and especially ischemia), which causes an immediate energy depletion and epithelial cell death (Vogt and Farber, 1968). After an injury, cell proliferation is necessary for epithelial repair (Humphreys et al., 2008). Surprisingly, an increase in cell proliferation caused by the inactivation of the anti-proliferative factors p53 and p21 was shown to worsen the lesions caused by an episode of ischemia-reperfusion in the kidney (Li et al., 2015; Megyesi et al., 2001; Sutton et al., 2013). Similarly, we found an association between increased proliferation, ATP depletion and increased cell death in renal epithelial cells in which Nupr1 (Nuclear Protein 1) was inactivated (Galichon et al., 2017). Nupr1 is a downstream effector of ATF4, a master regulator of endoplasmic reticulum stress, the activation of which leads to excessive protein synthesis, causing energy depletion and cell death (Han et al., 2013). In tumors, there is strong evidence that proliferation is associated with high energy production involving both oxidative phosphorylation and glycolysis a phenomenon known as the Warburg effect (Liberti and Locasale, 2016). Taken together, these studies suggest that an increase in cell proliferation in conditions of stress might cause critical energy depletion due to simultaneous enhanced energy needs for both cell maintenance and biosynthetic processes, thus resulting in cell death if a critical energy threshold is not maintained (Lunt and Vander Heiden, 2011). 


\section{Intercellular and intracellular variations in energy metabolism}

Our observations change the understanding of energy variations in proliferating cells (Figure 4c). Previous experiments were limited by the fact that timed bulk cell analysis did not allow the study of the ATP/ADP trajectories of single-cells within the same extracellular environment. We show by single cell analysis that the progressive increase in ATP/ADP ratio in proliferating cells results from increasing confluence and contact inhibition proliferation arrest, whereas proliferation itself (at the single-cell level) is associated with decreased ATP/ADP ratio. In addition, we show that within proliferating cells, the ATP/ADP ratio oscillates with the cell cycle. The intracellular energy level not only varies during the cell cycle but also depends on the proliferation rate. The live monitoring of the ATP/ADP ratio in single cells demonstrates transient physiological decreases in ATP/ADP in proliferating cells. Although healthy under basal conditions, these proliferating cells with lower ATP/ADP ratio were more vulnerable to a superimposed injury than non-proliferating cells. These oscillations in ATP/ADP ratio are reminiscent of cyclic variations of the TCA cycle flux described by Ahn and colleagues (Ahn et al., 2017), and this is in keeping with our data in vivo showing that oxidative phospholylation transcripts are overexpressed in proliferating cells.

Mendelsohn et al. found that cell growth and ATP can follow dissociated patterns in a high throughput CRISPRi screen (Mendelsohn et al., 2018). We provide experimental evidence that intracellular energetic levels (ATP/ADP ratio) do not determine cell proliferation. Quite the opposite, we show that proliferation negatively impacts the ATP/ADP ratio. In addition, upon injury, the cell survival is improved by the inhibition of proliferation, whereas cells in which proliferation is stimulated are more sensitive to injury than non-proliferating ones. This is likely 
related to an accelerated energy consumption required for protein synthesis and other metabolic processes necessary for genetic amplification prior to cell division.

\section{Effect of proliferation on cell viability upon injury}

We found proliferation to be associated with chemosensitivity, as previously described (Ramaker et al., 2017; Chatterjee et al., 2015; Krtinic et al., 2018; Song et al., 2010) and provide experimental data to demonstrate the causal relationship between proliferation and sensitivity to cell death. Considering that cancer cells with a high intracellular ATP level are more resistant to cytotoxic chemotherapy (Zhou et al., 2012), slow proliferation might be a mechanism for cancer to resist to chemotherapy or ischemia by maintaining high intracellular ATP/ADP ratio. Similarly, inhibiting proliferation might be a protective to prevent lethal energy depletion in epithelial cells. This protective mechanism might be responsible for the protective effect of the extensively studied phenomenon of ischemic preconditioning (Lieberthal et al., 2016; Bon et al., 2012; Bonventre, 2002), where a mild and transient ischemia triggers cytoprotective pathways concomitant with transient cell cycle arrest (Nishioka et al., 2014; Sano et al., 2000). Among these pathways, p53, p21 and AMPK were shown to protect the kidneys from ischemiareperfusion injury (Lee et al., 2020) while suppressing cell proliferation (Motoshima et al., 2006; Jones et al., 2005).

However, cell proliferation is absolutely necessary to vital processes, e.g. epithelial proliferation following renal epithelial injury is known to be instrumental to organ repair (Humphreys et al., 2008). Proliferation demands a high energetic input, and oxidative phosphorylation is the most efficient pathway for energy production. In keeping with that, we found that proliferation is associated with increased expression of nucleus-encoded OXPHOS genes. We found that the kidneys with an unfavorable evolutive profile had a dissociated transcriptomic pattern (high metaPCNA with maintained expression of nucleus-encoded 
OXPHOS genes but decreased expression of mitochondrially-encoded OXPHOS genes). This is in keeping with recent studies showing that maintaining oxidative phosphorylation during and after acute kidney injury (AKI) is protective (Kang et al., 2015; Poyan Mehr et al., 2018; Tran et al., 2016), and other studies suggesting that energy-parcimonious approaches might mitigate renal damage at the early stage of acute kidney injury. Stabilization of the hypoxia inducible factor (HIF) shifts the energy metabolism to anaerobic glycolysis, inhibits cell proliferation(Gordan et al., 2007; Hubbi and Semenza, 2015; Koshiji et al., 2004) and protects against ischemic acute kidney injury (Schley et al., 2011; Wang et al., 2006; Shu et al., 2019).

In sum, we show that proliferation is an energy demanding process impairing the cellular ability to cope with a toxic or ischemic injury. We postulate that interventions to mitigate proliferation and restore energy production can enhance cell survival and organ recovery (Figure 5). 


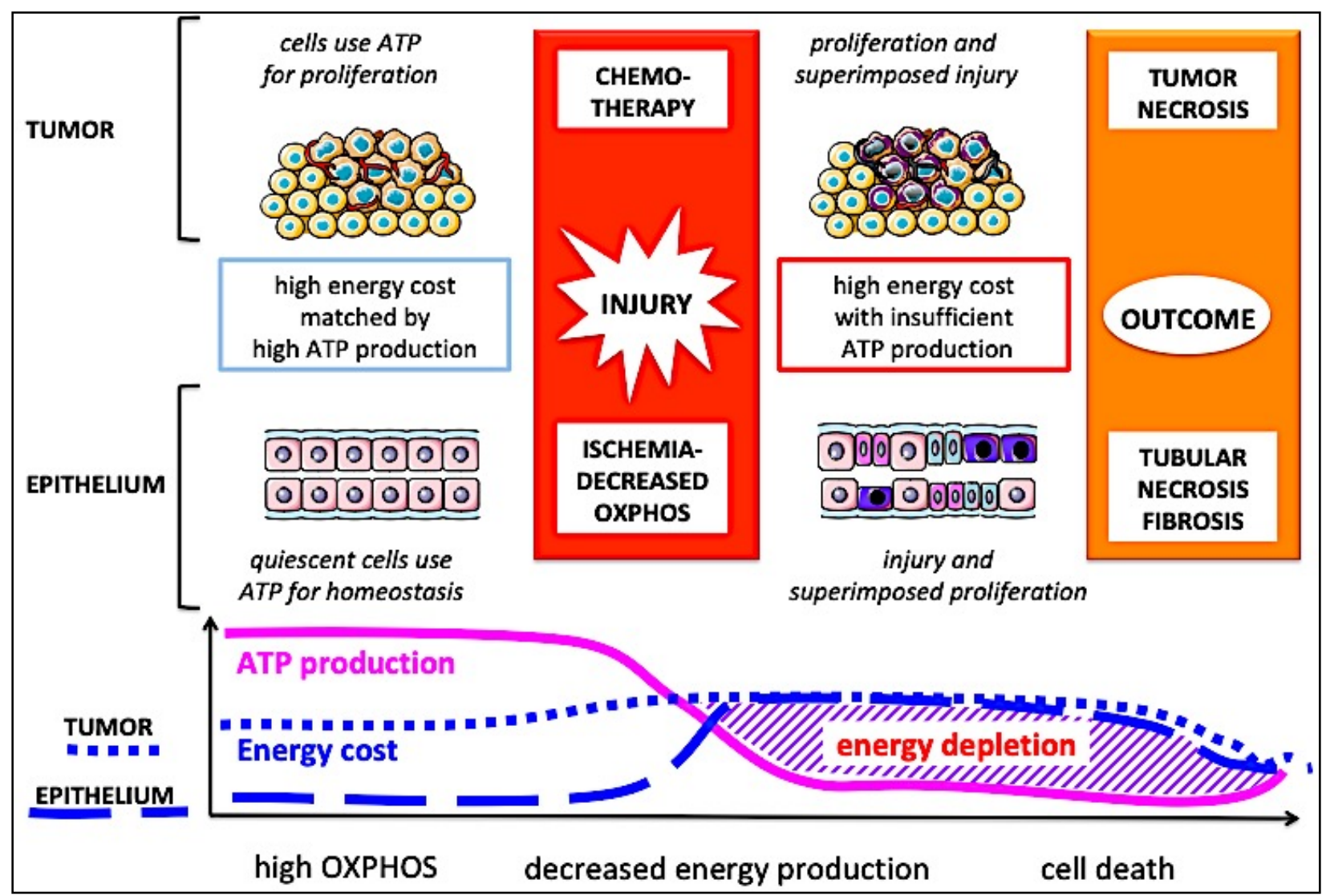

Figure 5. Impact of energy metabolism changes leading to cellular injury in proliferative cancers treated with chemotherapy and in injured kidneys. 


\section{METHODS}

\section{Cell cultures and pharmaceutical inhibitors}

HK2 cells are immortalized human renal proximal tubular cells cultured in DMEM with 10\% fetal bovine serum. The experiments were conducted in Leibovitz's L-15 medium with no phenol red (Fisher, \#21083027). L-15 was specifically designed for renal primary cell cultures. It provides a stable physiological $\mathrm{pH}$ without $\mathrm{CO}_{2}$ supplementation, as it is buffered with nonbicarbonate ions. Pharmaceutical inhibitors were purchased from Selleckchem: tenovin-1

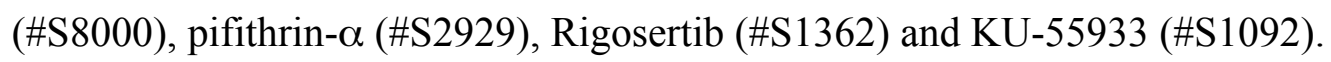

\section{Viability experiments}

Cells were grown in DMEM with $10 \%$ fetal bovine serum in the presence or absence of puromycin $(2 \mu \mathrm{g} / \mathrm{mL})$. Energy depletion was achieved in cells grown in Leibovitz's L-15 medium without glucose supplementation and hypoxia obtained by applying a 100\% N2 atmosphere (ref 26700 from Air products) in a airtight chamber (ref 27310 and 27311 from Stemcell) during 24h, according to the manufacturer's instructions. The control cells were grown in Leibovitz's L-15 medium with $4.5 \mathrm{~g} / \mathrm{dL}$ glucose supplementation under ambient atmosphere. Flow cytometry analysis for assessment of dead cells was performed with the fixable viability dye Viobility 405/452 Fixable Dye (Miltenyi Biotec $\left.{ }^{\circledR}\right)$. Viobility dyes react with amine groups of proteins and are non-toxic. In dead cells, intracellular proteins are stained, resulting in an increase in fluorescence. 
ATP/ADP bioluminescence assay

ATP and ADP measurements were performed with the ApoSENSOR ADP/ATP ratio assay (Enzo Life Sciences), in accordance with the manufacturer's instructions.

ATP/ADP ratio live imaging

PercevalHR was used to study the ATP/ADP ratio in live individual cells in real-time experiments, spanning multiple cell cycles. Using lentiviral transformation, we generated proximal tubular cells with stable expression of PercevalHR as well as pHRed, in order to adjust the PercevalHR signal on $\mathrm{pH}$ variations (Tantama et al., 2013). ATP/ADP ratio and $\mathrm{pH}$ were assessed by dual signal acquisition for PercevalHR (ex 436/495; em 540) and pHRed (ex 436/550; em 640), followed by ratiometric normalization using the Ratio Plus plugin in Fiji (Schindelin et al., 2012). PercevalHR (ATP/ADP ratio) was further corrected for $\mathrm{pH}$ by normalization on pHRed (Tantama et al., 2013), using the Ratio Plus plugin. 


\section{Bioinformatic analysis}

ATP/ADP ratio. The Fiji Trackmate plugin was used to monitor single-cell ATP/ADP ratio over time, and to determine the time of cytokinesis for each cell (Tinevez et al., 2017). Briefly, every cell was assigned to a single trajectory at a specific timepoint, with a corresponding ATP/ADP value. The times of cytokinesis were noted for each cell trajectory. ATP/ADP values were represented on the y-axis as mean+/-standard error, and time was represented on the x-axis in hours from either the start of the experiment or from the time of cytokinesis.

\section{Gene profiling experiments}

Computation of proliferation index, overall OXPHOS index and mitochondrial index. We used a previously published and validated method to compute a proliferation index as the median value of a list of proliferation associated genes (Venet et al., 2011). Similarly, we computed the overall OXPHOS index as the median value of the genes listed in the human OXPHOS pathway of the (Kyoto Encyclopedia of Genes and Genomes database (Kanehisa and Goto, 2000). We performed the same computation on the subset of mitochondria-encoded genes to obtain the mitochondrial OXPHOS index.

Correlation analysis of proliferation with chemosensitivity. We performed a Kendall's test to compute the correlation (correlation coefficient $\mathrm{k}$ and $\mathrm{p}$-value) of half maximal inhibitory concentretion (IC50) value with the proliferation index for every compound tested.

Pathway analysis of proliferation associated genes. We performed a Kendall's test to compute the correlation (correlation coefficient $\mathrm{k}$ and $\mathrm{p}$-value) of every gene expression value with the proliferation index. The correlations' p-values and coefficient were then processed with the LrPath web-based software allowing a logistic regression pathway analysis to identify the gene sets enriched in genes with proliferation-correlated expression levels. Three prespecified geneset 
for energy-producing pathways were analyzed: "oxidative phosphorylation", "Tricarboxylic Acid cycle", and "glycolysis". P-values and Odds Ratios were computed as indicators of the significance and the magnitude of the enrichment of these gene sets in genes with proliferationcorrelated expression values.

\section{Statistical analysis}

Statistical analyses were conducted using JMP 11 (SAS). Log-transformation was performed to obtain normal distribution, when necessary. Proportions, 95\% confidence interval and p-values were computed using the exact binomial test. Correlations were evaluated using Kendall's test for non-parametric values. P-values were considered significant when $<0.05$. Comparison between groups were considered significant when the p-value was $<0.05$ using a Wilcoxon rank-test. Differences in ATP/ADP changes between treatment groups over time were assessed by analysis of variance using a standard least square model. 


\section{ACKOWLEGMENTS}

The results shown here are in part based upon data generated by the TCGA Research Network: https://www.cancer.gov/tcga. PG received support from Monahan Foundation, Fondation pour la Recherche Médicale, Groupe Pasteur Mutualité , Société Francophone de Transplantation, Arthur Sachs fellowship, Philippe Foundation, Fulbright Scholarship, ATIP Avenir program. JVB was supported by grants from the National Institute of Health/NIDDK 2R01DK072381, R37DK039773 and UH3 TR002155.

\section{SUPPLEMENTAL MATERIAL:}

\begin{tabular}{|lll|}
\hline $\begin{array}{l}\text { drug (proliferation correlation } \\
\text { with chemosensitivity) }\end{array}$ & \multicolumn{1}{c}{ target } & \multicolumn{1}{c}{ pathway } \\
NPK76-II-72-1 (+) & PLK3 & Cell cycle \\
venotoclax (+) & Bcl2 & apoptose, MTP inhibitor \\
methotrexate (+) & antimetabolite & DNA replication \\
vorinostat (+) & HDACi & histone acetylation \\
ACY-1215 (+) & HDAC6i & histone acetylation \\
navitoclax (+) & Bcl2 & apoptose \\
daporinad (+) & NAMPT & metabolism \\
PHA-793887 (+) & CDK2/7/5 & Cell cycle \\
Ara-G (+) & antimetabolite & DNA synthesis \\
tivozanib (+) & VEGFR1/2/3 & RTK signalling \\
GSK1070916 (+) & AURKA/C & mitose \\
brivanib, BMS-540215 (+) & VEGFR/PDGFR & RTK signalling \\
kb NB 142-70 (+) & PKD & other \\
TL-2-105 (+) & CRAF inhibitor & ERK/MAPK \\
XMD13-2 (+) & RIPK1 & apoptosis \\
T0901317 (+) & LXR,FXR & other \\
alisertib (+) & AURKA & mitose \\
trametinib (-) & MEK1/2 & ERK/MAPK \\
refametinib (-) & MEK1/3 & ERK/MAPK \\
selumetinib (-) & MEK1/4 & ERK/MAPK \\
\hline
\end{tabular}

Supplemental Table1. Top 20 Drugs with the highest correlation of chemosensitivity with proliferation, with their molecular targets and biological pathways. In blue, drugs that are more toxic in proliferating cells, and in red, drugs that are less toxic in proliferating cells. MTP: mitochondrial transition pore. 


\begin{tabular}{|c|c|c|}
\hline TCGA & R (pval) & genes significantly associated with proliferation $(p<0.05)$ \\
\hline OXPHOS & $\begin{array}{l}07 \\
(3 e-10)\end{array}$ & $\begin{array}{l}\text { SDHB, SDHD, MT-ND1, ATP5F1B, MT-ATP6, NDUFA13, SDHA, MT-ND2, TCIRG1, MT-ND4, SDHC, MT-CO1, } \\
\text { MT-CO2, MT-ND3, PPA1, COX4I1, NDUFS3, NDUFS1, CYC1, ATP6VOC, MT-CYB, ATP5F1C, ATP5PF, NDUFS2, } \\
\text { NDUFV1, UQCRB, UQCRC1, ATP5PO, ATP6V1B2, ATP6V1A, COX17, ATP6V1H, MT-CO3, ATP6AP1, } \\
\text { ATP6V1E1, COX5B, NDUFS7, ATP5PB, NDUFA4, ATP6V0D1, ATP6V0A1, NDUFA1, COX7A1, NDUFB11, } \\
\text { NDUFB6, NDUFS8, ATP6V1C1, UQCRFS1, COX6B1, ATP5PD, NDUFA10, ATP6V1G1, COX4I2, ATP5F1D, } \\
\text { COX5A, COX7A2, ATP5MF, NDUFB9, PPA2, COX7B, ATP5F1E, ATP5MG, MT-ATP8, NDUFB8, NDUFV3, } \\
\text { COX6C, ATP5ME, COX6A1, NDUFAB1, COX7A2L, NDUFA2, NDUFA8, NDUFS5, ATP5MC1, ATP6V1F, UQCRH, } \\
\text { NDUFB10, ATP6V1D, ATP6VOB, NDUFC2, NDUFS6, NDUFA4L2, LHPP, NDUFA12, NDUFA6, ATP6V0E1, } \\
\text { ATP5MC2, COX8A, NDUFB7, UQCRQ, COX7C, UQCR10, MT-ND4L, NDUFB5, NDUFB4, ATP5MC3, NDUFB3, } \\
\text { NDUFA3, NDUFA11, NDUFB1, NDUFB2, NDUFC1, UQCR11 }\end{array}$ \\
\hline TCA & & $\begin{array}{l}\text {, PCK1, SDHC, DLD, PC, ACO2, DLAT, MDH2, CS, } \\
\text { GDHL, IDH3G }\end{array}$ \\
\hline Glyc & \begin{tabular}{|l|}
1.001 \\
$(5.70 \mathrm{e}-1)$
\end{tabular} & $\begin{array}{l}\text { ALDH2, PKM, ADH1B, GAPDH, LDHA, ENO1, ADH1C, HK2, PGK1, PDHA1, GPI, ALDOA, HK1, FBP1, TPI1, } \\
\text { LDHB, ALDH7A1, ALDH1A3, ALDH3A2, PCK1, PFKM, DLD, ADH5, PFKP, PGAM1, PFKL, DLAT, ALDH1B1, } \\
\text { ADH1A, PCK2, AKR1A1, ACSS2, BPGM, ALDH9A1, PGM2, GALM, ALDH3B1, ADPGK, ACSS1, ALDH3B2 }\end{array}$ \\
\hline reperfusion & OR(pval) & genes significantly associated with proliferation $(p<0.05)$ \\
\hline OXPHOS & $\begin{array}{l}0.542 \\
(2.75 e-22)\end{array}$ & $\begin{array}{l}\text { ATP5F1A, NDUFA13, MT-ND2, MT-ND4, MT-ND5, MT-CO1, MT-CO2, MT-ND3, ATP6VOA2, COX4I1, } \\
\text { NDUFS1, ATP6V0A4, MT-ND6, MT-CYB, ATP5F1C, NDUFS2, UQCRC2, ATP5PO, MT-CO3, ATP6AP1, } \\
\text { NDUFA10, NDUFB9, PPA2, MT-ATP8, COX15, ATP5MC2, COX11, MT-ND4L, NDUFA11 } \\
\text { MT-ND3, ATP6VOA2, COX4I1, NDUFS1, ATP6V0A4, MT-ND6, MT-CYB, ATP5F1C, NDUFS2, UQCRC2 } \\
\end{array}$ \\
\hline TCA & 00 & FH, PDHA1, ACLY, PCK1, DLD, PC, PDHB, MDH2, CS, IDH3B, IDH3G \\
\hline & $\begin{array}{l}0.869 \\
(7.61 \mathrm{e}-2)\end{array}$ & GAM1, PDHB, ALDOC, BPGM, HKDC1, PGM2, ADPGK, ACSS1 \\
\hline
\end{tabular}

Supplemental Table 2. Metabolic pathways and genes associated with proliferation in tumors (TCGA) and in injured kidneys (reperfusion). OXPHOS: oxidative phosphorylation pathway; TCA: tricarboxyclic acid cycle, OR: Odds Ratio for genes which expression values are correlated with the proliferation index to belong to the tested pathways (versus non correlated genes). 


\begin{tabular}{|c|c|c|c|}
\hline pathway & $\begin{array}{l}\text { recovery OR ( } p \\
\text { value) }\end{array}$ & $\begin{array}{l}\text { transitional OR ( } p \\
\text { value) }\end{array}$ & CKD OR ( $p$ value) \\
\hline OXPHOS & $0.297(9.75 e-22)$ & $0.201(1.28 e-15)$ & 0.207 (3.69e-41) \\
\hline$T C A$ & $0.311(7.06 e-8)$ & $1.210(5.27 \mathrm{e}-1)$ & $0.460(8.22 e-6)$ \\
\hline Glycolysis & $0.423(7.25 e-6)$ & 0.892 (6.73e-1) & $0.511(2.2 e-6)$ \\
\hline
\end{tabular}

Supplemental Table 3. Metabolic pathways and genes associated with proliferation in transplanted kidneys with different evolutive profiles: recovery, transition to chronic kidney disease (transitional), established chronic kidney disease (CKD). OXPHOS: oxidative phosphorylation pathway; TCA: tricarboxyclic acid cycle, OR: Odds Ratio for genes which expression values are correlated with the proliferation index to belong to the tested pathways (versus non correlated genes). 


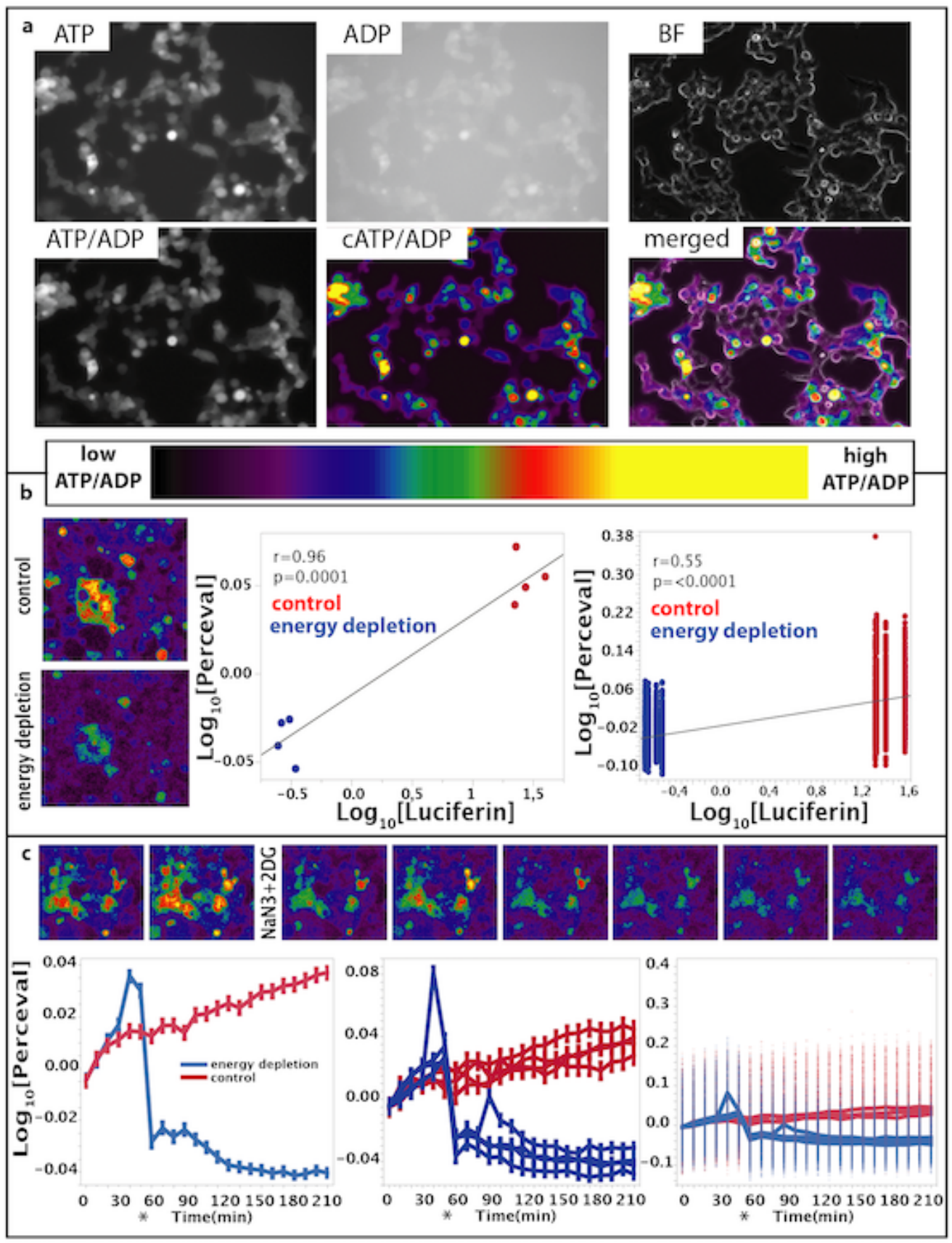

\section{Suppl Figure 1: Monitoring of intracellular ATP/ADP ratio.}

a. Fluorescence imaging of ATP-bound PercevalHR (ATP), ADP-bound PercevalHR (ADP), brightfield image $(\mathrm{BF}), 2 \mathrm{D}$-representation of the ATP/ADP ratio in black and white (ATP/ADP) and using artificial colors (cATP/ADP), and the cATP/ADP image merged with the brightfield image (merged). b. Comparison of the PercevalHR and Luciferin based assay for ATP/ADP quantification. c. Real time single cell ATP/ADP monitoring with PercevalHR. Left: each line represents the mean ATP/ADP value of cells from each condition (control vs energy depletion). Middle: each line represents the mean ATP/ADP value of the cells from a single well. Right: ATP/ADP values of individual cells represented by dots. The $\left\langle{ }^{*}\right\rangle$ sign indicates the time when the cells were treated with $2 \mathrm{DG}$ and $\mathrm{NaN}_{3}$ for energy depletion. 


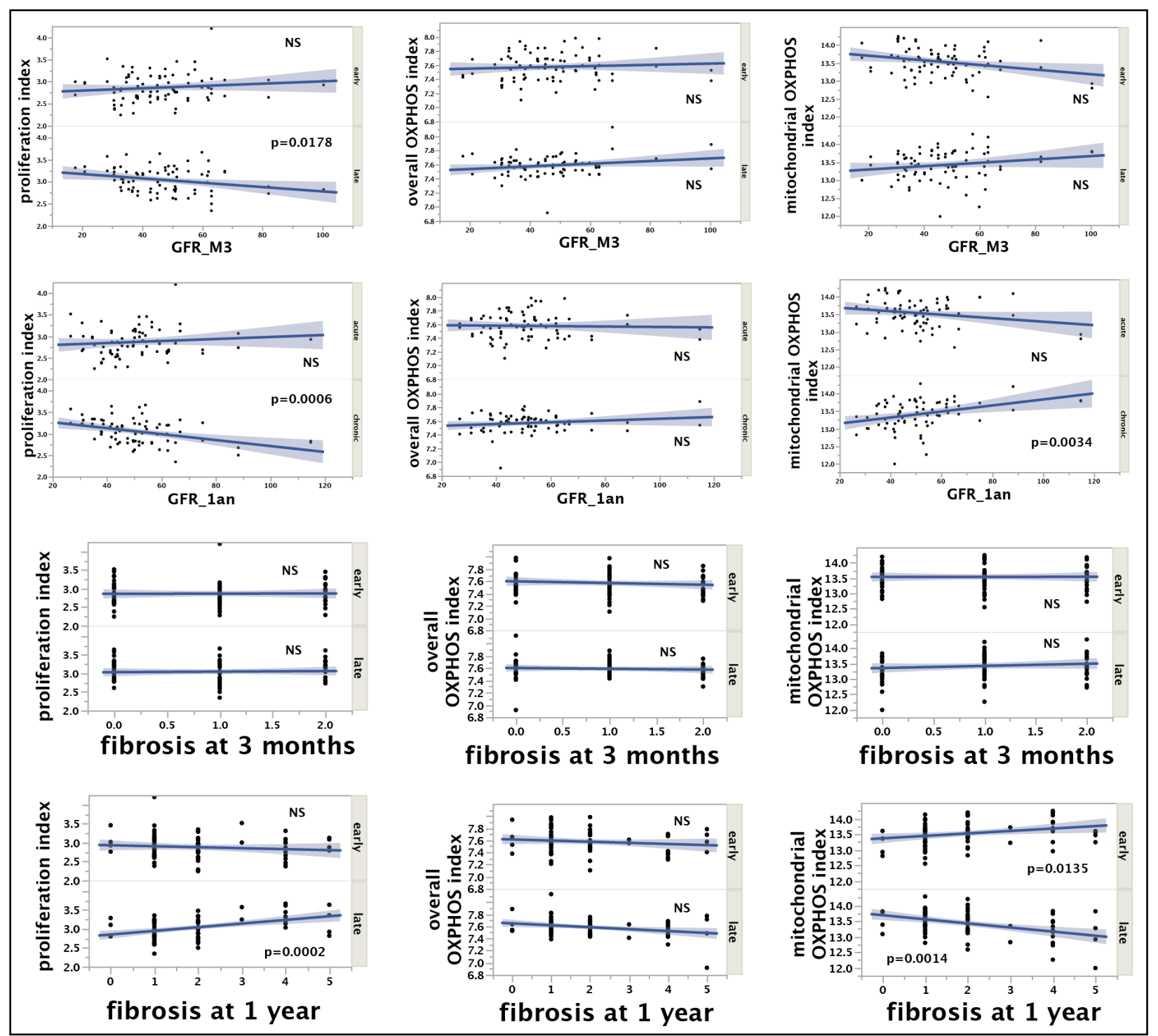

Suppl Figure 2. Association of the proliferation index and the OXPHOS indexes with long term outcomes. Proliferation index, overall OXPHOS index and mitochondrial OXPHOS index at acute (before transplantation and after reperfusion) and chronic time points (at 3 and 12 months after transplantation) differential correlations with 3 and 12 months glomerular filtration rate (GFR) and with 3 and 12 months kidney fibrosis., and the differential correlations of overall and mitochondrial OXPHOS with the proliferation index ( $p$-values calculated by Kendall correlation test for non-parametric values). 


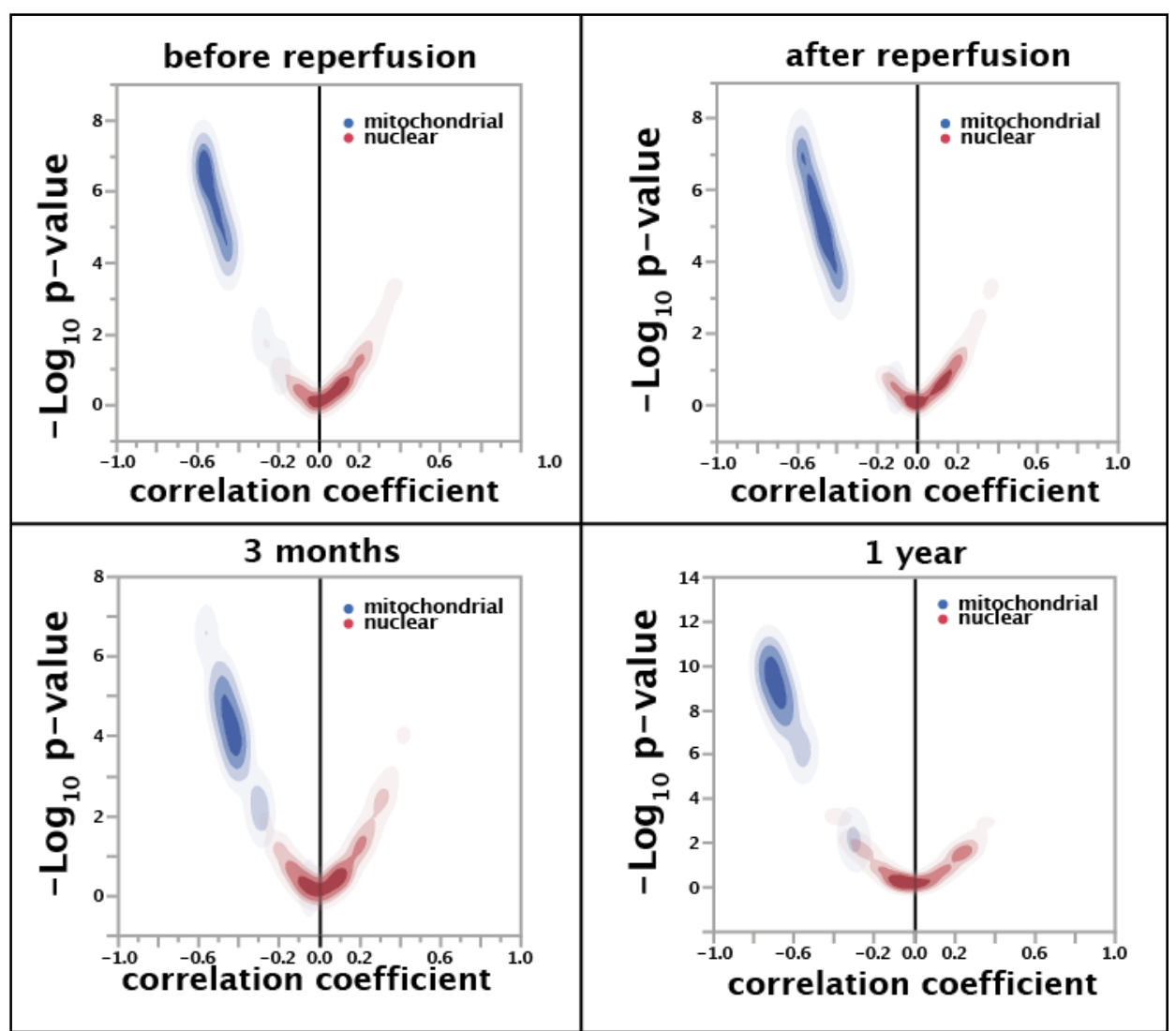

Suppl Figure 3. Volcano plot showing the significance of the correlation of the proliferation index with every expression value of the OXPHOS genes at the different time points after transplantation. Nucleus-encoded genes are in red and mitochondria-encoded genes are in red.

Fiji Jython script for single cell ratiometric analysis

Live PercevalHR videos 


\section{REFERENCES}

Ahn, E., Kumar, P., Mukha, D., Tzur, A., and Shlomi, T. (2017). Temporal fluxomics reveals oscillations in TCA cycle flux throughout the mammalian cell cycle. Mol Syst Biol 13, 953.

Atkinson, D. E. (1968). The energy charge of the adenylate pool as a regulatory parameter. Interaction with feedback modifiers. Biochemistry 7, 4030-4034.

Berg, J., Hung, Y. P., and Yellen, G. (2009). A genetically encoded fluorescent reporter of ATP:ADP ratio. Nat Methods 6, 161-166.

Bon, D., Chatauret, N., Giraud, S., Thuillier, R., Favreau, F., and Hauet, T. (2012). New strategies to optimize kidney recovery and preservation in transplantation. Nat Rev Nephrol 8 , 339-347.

Bonora, M., Patergnani, S., Rimessi, A., De Marchi, E., Suski, J. M., Bononi, A., Giorgi, C., Marchi, S., Missiroli, S., Poletti, F., Wieckowski, M. R., and Pinton, P. (2012). ATP synthesis and storage. Purinergic Signal 8, 343-357.

Bonventre, J. V. (2002). Kidney ischemic preconditioning. Curr Opin Nephrol Hypertens 11, 4348.

Chan, G. K., Kleinheinz, T. L., Peterson, D., and Moffat, J. G. (2013). A simple high-content cell cycle assay reveals frequent discrepancies between cell number and ATP and MTS proliferation assays. PLoS One 8 , e63583.

Chatterjee, D., Bal, A., Das, A., and Singh, G. (2015). Proliferation rate and breast cancer subtype, but not ALDH1 expression, predict pathological response to neoadjuvant chemotherapy in locally advanced breast cancer. Virchows Arch 467, 303-310.

Cippà, P. E., Sun, B., Liu, J., Chen, L., Naesens, M., and McMahon, A. P. (2018). Transcriptional trajectories of human kidney injury progression. JCI Insight 3, 
Crouch, S. P., Kozlowski, R., Slater, K. J., and Fletcher, J. (1993). The use of ATP bioluminescence as a measure of cell proliferation and cytotoxicity. J Immunol Methods 160, 8188.

Dixon, H. B. F. (1988). Teaching the role of AMP in metabolic control. Biochemical Education Biochem. Educ. 16, 214-217.

Fu, X. F., Yao, K., Du, X., Li, Y., Yang, X. Y., Yu, M., Li, M. Z., and Cui, Q. H. (2016). PGC-1 $\alpha$ regulates the cell cycle through ATP and ROS in CH1 cells. J Zhejiang Univ Sci B 17, 136-146. Galichon, P., Bataille, A., Vandermeersch, S., Wetzstein, M., Xu-Dubois, Y. C., Legouis, D., Hertig, A., Buob, D., Placier, S., Bigé, N., Lefevre, G., Jouanneau, C., Martin, C., Iovanna, J. L., and Rondeau, E. (2017). Stress Response Gene Nupr1 Alleviates Cyclosporin A Nephrotoxicity In Vivo. J Am Soc Nephrol 28, 545-556.

Gordan, J. D., Thompson, C. B., and Simon, M. C. (2007). HIF and c-Myc: sibling rivals for control of cancer cell metabolism and proliferation. Cancer Cell 12, 108-113.

Han, J., Back, S. H., Hur, J., Lin, Y. H., Gildersleeve, R., Shan, J., Yuan, C. L., Krokowski, D., Wang, S., Hatzoglou, M., Kilberg, M. S., Sartor, M. A., and Kaufman, R. J. (2013). ER-stressinduced transcriptional regulation increases protein synthesis leading to cell death. Nat Cell Biol $15,481-490$.

Hardie, D. G. (2011). AMP-activated protein kinase--an energy sensor that regulates all aspects of cell function. Genes \&amp; Development 25, 1895-1908.

Hardie, D. G., Ross, F. A., and Hawley, S. A. (2012). AMP-activated protein kinase: a target for drugs both ancient and modern. Chem Biol 19, 1222-1236.

Hubbi, M. E., and Semenza, G. L. (2015). Regulation of cell proliferation by hypoxia-inducible factors. Am J Physiol Cell Physiol 309, C775-82. 
Humphreys, B. D., Valerius, M. T., Kobayashi, A., Mugford, J. W., Soeung, S., Duffield, J. S., McMahon, A. P., and Bonventre, J. V. (2008). Intrinsic Epithelial Cells Repair the Kidney after Injury. Cell Stem Cell 2, 284-291.

Izyumov, D. S., Avetisyan, A. V., Pletjushkina, O. Y., Sakharov, D. V., Wirtz, K. W., Chernyak, B. V., and Skulachev, V. P. (2004). Wages of fear": transient threefold decrease in intracellular ATP level imposes apoptosis. Biochim Biophys Acta 1658, 141-147.

Jones, R. G., Plas, D. R., Kubek, S., Buzzai, M., Mu, J., Xu, Y., Birnbaum, M. J., and Thompson, C. B. (2005). AMP-Activated Protein Kinase Induces a p53-Dependent Metabolic Checkpoint. Molecular Cell 18, 283-293.

Kanehisa, M., and Goto, S. (2000). KEGG: kyoto encyclopedia of genes and genomes. Nucleic Acids Res 28, 27-30.

Kang, H. M., Ahn, S. H., Choi, P., Ko, Y. A., Han, S. H., Chinga, F., Park, A. S., Tao, J., Sharma, K., Pullman, J., Bottinger, E. P., Goldberg, I. J., and Susztak, K. (2015). Defective fatty acid oxidation in renal tubular epithelial cells has a key role in kidney fibrosis development. Nat Med 21, 37-46.

Koshiji, M., Kageyama, Y., Pete, E. A., Horikawa, I., Barrett, J. C., and Huang, L. E. (2004). HIF-1alpha induces cell cycle arrest by functionally counteracting Myc. EMBO J 23, 1949-1956. Krtinic, D., Zivadinovic, R., Jovic, Z., Pesic, S., Mihailovic, D., Ristic, L., Cvetanovic, A., Todorovska, I., Zivkovic, N., Rankovic, G. N., Stokanovic, D., Zivadinovic, B., Trandafilovic, M., Apostolovic, M. A., Golubovic, M., and Zivadinovic, A. (2018). Significance of the Ki-67 proliferation index in the assessment of the therapeutic response to cisplatin-based chemotherapy in patients with advanced cervical cancer. Eur Rev Med Pharmacol Sci 22, 5149-5155. 
Lee, H., Zandkarimi, F., Zhang, Y., Meena, J. K., Kim, J., Zhuang, L., Tyagi, S., Ma, L., Westbrook, T. F., Steinberg, G. R., Nakada, D., Stockwell, B. R., and Gan, B. (2020). Energystress-mediated AMPK activation inhibits ferroptosis. Nat Cell Biol 22, 225-234.

Lelli, J. L., Becks, L. L., Dabrowska, M. I., and Hinshaw, D. B. (1998). ATP converts necrosis to apoptosis in oxidant-injured endothelial cells. Free Radic Biol Med 25, 694-702.

Li, Y., Liu, J., Li, W., Brown, A., Baddoo, M., Li, M., Carroll, T., Oxburgh, L., Feng, Y., and Saifudeen, Z. (2015). p53 enables metabolic fitness and self-renewal of nephron progenitor cells. Development 142, 1228-1241.

Liberti, M. V., and Locasale, J. W. (2016). The Warburg Effect: How Does it Benefit Cancer Cells. Trends Biochem Sci 41, 211-218.

Lieberthal, W., Tang, M., Lusco, M., Abate, M., and Levine, J. S. (2016). Preconditioning mice with activators of AMPK ameliorates ischemic acute kidney injury in vivo. Am J Physiol Renal Physiol 311, F731-F739.

Lunt, S. Y., and Vander Heiden, M. G. (2011). Aerobic glycolysis: meeting the metabolic requirements of cell proliferation. Annu Rev Cell Dev Biol 27, 441-464.

Maldonado, E. N., and Lemasters, J. J. (2014). ATP/ADP ratio, the missed connection between mitochondria and the Warburg effect. Mitochondrion 19, 78-84.

Marcussen, M., and Larsen, P. J. (1996). Cell cycle-dependent regulation of cellular ATP concentration, and depolymerization of the interphase microtubular network induced by elevated cellular ATP concentration in whole fibroblasts. Cell Motil Cytoskeleton 35, 94-99.

Megyesi, J., Andrade, L., Vieira, J. M., Safirstein, R. L., and Price, P. M. (2001). Positive effect of the induction of p21WAF1/CIP1 on the course of ischemic acute renal failure. Kidney Int 60 , 2164-2172. 
Mendelsohn, B. A., Bennett, N. K., Darch, M. A., Yu, K., Nguyen, M. K., Pucciarelli, D., Nelson, M., Horlbeck, M. A., Gilbert, L. A., Hyun, W., Kampmann, M., Nakamura, J. L., and Nakamura, K. (2018). A high-throughput screen of real-time ATP levels in individual cells reveals mechanisms of energy failure. PLoS Biol 16, e2004624.

Motoshima, H., Goldstein, B. J., Igata, M., and Araki, E. (2006). AMPK and cell proliferation-AMPK as a therapeutic target for atherosclerosis and cancer. J Physiol 574, 63-71.

Nishioka, S., Nakano, D., Kitada, K., Sofue, T., Ohsaki, H., Moriwaki, K., Hara, T., Ohmori, K., Kohno, M., and Nishiyama, A. (2014). The cyclin-dependent kinase inhibitor p21 is essential for the beneficial effects of renal ischemic preconditioning on renal ischemia/reperfusion injury in mice. Kidney Int 85, 871-879.

Poyan Mehr, A., Tran, M. T., Ralto, K. M., Leaf, D. E., Washco, V., Messmer, J., Lerner, A., Kher, A., Kim, S. H., Khoury, C. C., Herzig, S. J., Trovato, M. E., Simon-Tillaux, N., Lynch, M. R., Thadhani, R. I., Clish, C. B., Khabbaz, K. R., Rhee, E. P., Waikar, S. S., Berg, A. H., and Parikh, S. M. (2018). De novo NAD+ biosynthetic impairment in acute kidney injury in humans. Nat Med 24, 1351-1359.

Price, P. M., Megyesi, J., and Saf Irstein, R. L. (2004). Cell cycle regulation: repair and regeneration in acute renal failure. Kidney Int 66, 509-514.

Ramaker, R. C., Lasseigne, B. N., Hardigan, A. A., Palacio, L., Gunther, D. S., Myers, R. M., and Cooper, S. J. (2017). RNA sequencing-based cell proliferation analysis across 19 cancers identifies a subset of proliferation-informative cancers with a common survival signature. Oncotarget 8, 38668-38681.

Roger, S., Erwin, S., and Erwin, S. D. (1992). What is Life. 
Sano, K., Fujigaki, Y., Miyaji, T., Ikegaya, N., Ohishi, K., Yonemura, K., and Hishida, A. (2000). Role of apoptosis in uranyl acetate-induced acute renal failure and acquired resistance to uranyl acetate. Kidney Int 57, 1560-1570.

Schindelin, J., Arganda-Carreras, I., Frise, E., Kaynig, V., Longair, M., Pietzsch, T., Preibisch, S., Rueden, C., Saalfeld, S., Schmid, B., Tinevez, J. Y., White, D. J., Hartenstein, V., Eliceiri, K., Tomancak, P., and Cardona, A. (2012). Fiji: an open-source platform for biological-image analysis. Nat Methods 9, 676-682.

Schley, G., Klanke, B., Schodel, J., Forstreuter, F., Shukla, D., Kurtz, A., Amann, K., Wiesener, M. S., Rosen, S., Eckardt, K. U., Maxwell, P. H., and Willam, C. (2011). Hypoxia-inducible transcription factors stabilization in the thick ascending limb protects against ischemic acute kidney injury. J Am Soc Nephrol 22, 2004-2015.

Sholl-Franco, A., Fragel-Madeira, L., Macama, A. C., Linden, R., and Ventura, A. L. (2010). ATP controls cell cycle and induces proliferation in the mouse developing retina. Int $\mathrm{J}$ Dev Neurosci 28, 63-73.

Shu, S., Wang, Y., Zheng, M., Liu, Z., Cai, J., Tang, C., and Dong, Z. (2019). Hypoxia and Hypoxia-Inducible Factors in Kidney Injury and Repair. Cells 8 ,

Song, D., Liu, X., Liu, R., Yang, L., Zuo, J., and Liu, W. (2010). Connexin 43 hemichannel regulates $\mathrm{H} 9 \mathrm{c} 2$ cell proliferation by modulating intracellular ATP and $[\mathrm{Ca} 2+]$. Acta Biochimica et Biophysica Sinica 42, 472-482.

Sutton, T. A., Hato, T., Mai, E., Yoshimoto, M., Kuehl, S., Anderson, M., Mang, H., Plotkin, Z., Chan, R. J., and Dagher, P. C. (2013). p53 is renoprotective after ischemic kidney injury by reducing inflammation. J Am Soc Nephrol 24, 113-124. 
Tantama, M., Martínez-François, J. R., Mongeon, R., and Yellen, G. (2013). Imaging energy status in live cells with a fluorescent biosensor of the intracellular ATP-to-ADP ratio. Nat Commun 4, 2550.

Tarasov, A. I., Griffiths, E. J., and Rutter, G. A. (2012). Regulation of ATP production by mitochondrial $\mathrm{Ca}(2+)$. Cell Calcium 52, 28-35.

Tinevez, J. Y., Perry, N., Schindelin, J., Hoopes, G. M., Reynolds, G. D., Laplantine, E., Bednarek, S. Y., Shorte, S. L., and Eliceiri, K. W. (2017). TrackMate: An open and extensible platform for single-particle tracking. Methods 115, 80-90.

Tran, M. T., Zsengeller, Z. K., Berg, A. H., Khankin, E. V., Bhasin, M. K., Kim, W., Clish, C. B., Stillman, I. E., Karumanchi, S. A., Rhee, E. P., and Parikh, S. M. (2016). PGC1 $\alpha$ drives NAD biosynthesis linking oxidative metabolism to renal protection. Nature 531, 528-532.

Vander Heiden, M. G., Cantley, L. C., and Thompson, C. B. (2009). Understanding the Warburg effect: the metabolic requirements of cell proliferation. Science 324, 1029-1033.

Veech, R. L., Lawson, J. W., Cornell, N. W., and Krebs, H. A. (1979). Cytosolic phosphorylation potential. J Biol Chem 254, 6538-6547.

Venet, D., Dumont, J. E., and Detours, V. (2011). Most random gene expression signatures are significantly associated with breast cancer outcome. PLoS Comput Biol 7, e1002240.

Verrax, J., Dejeans, N., Sid, B., Glorieux, C., and Calderon, P. B. (2011). Intracellular ATP levels determine cell death fate of cancer cells exposed to both standard and redox chemotherapeutic agents. Biochem Pharmacol 82, 1540-1548.

Vogt, M. T., and Farber, E. (1968). On the molecular pathology of ischemic renal cell death. Reversible and irreversible cellular and mitochondrial metabolic alterations. Am J Pathol 53, 126. 
Wang, J., Biju, M. P., Wang, M. H., Haase, V. H., and Dong, Z. (2006). Cytoprotective effects of hypoxia against cisplatin-induced tubular cell apoptosis: involvement of mitochondrial inhibition and p53 suppression. J Am Soc Nephrol 17, 1875-1885.

Wang, X., Li, L., Guan, R., Zhu, D., Song, N., and Shen, L. (2017). Emodin Inhibits ATPInduced Proliferation and Migration by Suppressing P2Y Receptors in Human Lung Adenocarcinoma Cells. Cell Physiol Biochem 44, 1337-1351.

Yang, W., Soares, J., Greninger, P., Edelman, E. J., Lightfoot, H., Forbes, S., Bindal, N., Beare, D., Smith, J. A., Thompson, I. R., Ramaswamy, S., Futreal, P. A., Haber, D. A., Stratton, M. R., Benes, C., McDermott, U., and Garnett, M. J. (2013). Genomics of Drug Sensitivity in Cancer (GDSC): a resource for therapeutic biomarker discovery in cancer cells. Nucleic Acids Res 41, D955-61.

Zhou, Y., Tozzi, F., Chen, J., Fan, F., Xia, L., Wang, J., Gao, G., Zhang, A., Xia, X., Brasher, H., Widger, W., Ellis, L. M., and Weihua, Z. (2012). Intracellular ATP levels are a pivotal determinant of chemoresistance in colon cancer cells. Cancer Res 72, 304-314. 